ARTICLE

Received 27 May 2014 | Accepted 14 Dec 2014 | Published 21 Jan 2015

DOl: 10.1038/ncomms7121 OPEN

\title{
The adhesion G protein-coupled receptor GPR56 is a cell-autonomous regulator of oligodendrocyte development
}

\author{
Stefanie Giera ${ }^{1, \star}$, Yiyu Deng ${ }^{1, \star,}$, , Rong Luo ${ }^{1, \star}$, Sarah D. Ackerman ${ }^{2}$, Amit Mogha ${ }^{2}$, Kelly R. Monk ${ }^{2,3}$, \\ Yanqin Ying ${ }^{1}$, Sung-Jin Jeong ${ }^{1, \dagger}$, Manabu Makinodan ${ }^{4,5}$, Allison R. Bialas ${ }^{4,5}$, Bernard S. Chang 6 , \\ Beth Stevens 4,5 , Gabriel Corfas ${ }^{4,5, \dagger}$ \& Xianhua Piao ${ }^{1}$
}

Mutations in GPR56, a member of the adhesion G protein-coupled receptor family, cause a human brain malformation called bilateral frontoparietal polymicrogyria (BFPP). Magnetic resonance imaging (MRI) of BFPP brains reveals myelination defects in addition to brain malformation. However, the cellular role of GPR56 in oligodendrocyte development remains unknown. Here, we demonstrate that loss of Gpr56 leads to hypomyelination of the central nervous system in mice. GPR56 levels are abundant throughout early stages of oligodendrocyte development, but are downregulated in myelinating oligodendrocytes. Gpr56-knockout mice manifest with decreased oligodendrocyte precursor cell (OPC) proliferation and diminished levels of active RhoA, leading to fewer mature oligodendrocytes and a reduced number of myelinated axons in the corpus callosum and optic nerves. Conditional ablation of Gpr56 in OPCs leads to a reduced number of mature oligodendrocytes as seen in constitutive knockout of Gpr56. Together, our data define GPR56 as a cell-autonomous regulator of oligodendrocyte development.

\footnotetext{
${ }^{1}$ Division of Newborn Medicine, Department of Medicine, Boston Children's Hospital and Harvard Medical School, Boston, Massachusetts 02115, USA.

${ }^{2}$ Department of Developmental Biology, Washington University School of Medicine, St Louis, Missouri 63110, USA. ${ }^{3}$ Hope Center for Neurological Disorders, Washington University School of Medicine, St Louis, Missouri 63110, USA. ${ }^{4}$ F.M. Kirby Neurobiology Center, Children's Hospital, Harvard Medical School, Boston, Massachusetts 02115, USA. ${ }^{5}$ Department of Neurology, Harvard Medical School, Boston, Massachusetts 02115, USA. ${ }^{6}$ Comprehensive Epilepsy Center, Department of Neurology, Beth Israel Deaconess Medical Center and Harvard Medical School, Boston, Massachusetts 02115, USA. * These authors contributed equally to this work. †Present addresses: Department of Emergency \& Critical Care Medicine, Guangdong General Hospital, Guangdong Academy of Medical Sciences, Guangzhou 510080, PR China (Y. D.); Convergence Brain Research Department, Korea Brain Research Institute (KBRI), Daegu 701-300, South Korea (S.-J.J.); Kresge Hearing Research Institute, Department of Otolaryngology - Head and Neck Surgery, University of Michigan, Ann Arbor, Michigan 48109, USA (G.C.). Correspondence and requests for materials should be addressed to X.P. (email: xianhua.piao@childrens.harvard.edu).
} 
M yelin, the multilayered glial membrane surrounding axons, is paramount to axon conductivity and health in the vertebrate nervous system. In the central nervous system (CNS), myelin is formed by specialized glial cells called oligodendrocytes $(\mathrm{OLs})^{1,2}$. The molecular mechanisms that govern OL development are only beginning to be elucidated, yet several studies have implicated extracellular matrix (ECM) proteins and their receptors as important extrinsic regulators of these processes ${ }^{3-6}$. The family of adhesion $G$ protein-coupled receptors (aGPCRs) is a major class of ECM receptors that mediates cell-matrix interactions, and recent reports implicate aGPCRs as important regulators of myelination ${ }^{7-11}$. Thus, we hypothesize that these receptors could play a major role during OL development and myelination.

Mutations in GPR56, a member of the aGPCR family, cause a devastating human brain malformation called bilateral frontoparietal polymicrogyria (BFPP), in which the normal convoluted brain surface is replaced by numerous small gyri ${ }^{12,13}$. In addition to this cortical defect, BFPP brains also show signs of myelination abnormalities, namely reduced white matter volume and signal changes on MRIs ${ }^{12,13}$, suggesting that GPR56 is important for myelination. However, it is not clear whether this myelination abnormality results from abnormal neuronal development, an intrinsic defect in OLs, or both.

Previous biochemical studies demonstrate that BFPPassociated mutations result in loss of GPR56 function attributable to aberrant processing/trafficking of the protein or loss of its ligand binding ability ${ }^{14-18}$. Consistent with these findings, Gpr56knockout mice exhibit forebrain and cerebellar defects similar to those seen in BFPP ${ }^{19,20}$. However, the cellular roles of GPR56 in CNS myelination remain unclear. A recent study shows that GPR56 regulates neural stem cell proliferation ${ }^{21}$, but its role in OL development remains unknown.

In light of the severe CNS myelination abnormalities seen in BFPP patients, we studied the cerebral white matter of Gpr56-knockout mice. We used histological, cellular and molecular approaches to characterize the defects caused by loss of GPR56. Here, we show that GPR56 levels are the highest in OL precursor cells (OPCs), and that GPR56 levels decline as OLs develop. We demonstrate that GPR56 is required for proper CNS myelination by controlling OPC proliferation, and that this is likely mediated by RhoA activation. Finally, we demonstrate that the defects caused by loss of GPR56 arise from a cell-autonomous defect in OL-lineage cells. Together, these studies define GPR56 as a novel regulator of OL development.

\section{Results}

Loss of GPR56 results in CNS myelination defects. CNS white matter abnormalities, manifesting as bilateral foci of T2 signal intensity change within the cerebral white matter on brain MRIs (Supplementary Fig. 1, right panel), is one of the hallmarks of BFPP brains ${ }^{12,13,22,23}$. To begin to investigate the role of GPR56 in CNS myelination, we examined the myelination status of Gpr56knockout mouse brains ${ }^{20,24}$. We have previously shown that there is no brain phenotype associated with GPR56 heterozygous status in both humans and mice ${ }^{12,13,19,20}$. Furthermore, we observed the same number of EGFP ${ }^{+}$OLs in the CC of $\mathrm{Gpr}^{2} 6^{+/+}$and Gpr56 $6^{+1-}$ mice at P28 (Supplementary Fig. 2), confirming that loss of one allele of Gpr56 causes no OL phenotype. Thus, we used Gpr56 ${ }^{+1-}$ mice as littermate controls whenever possible to conserve animals. Staining for FluoroMyelin, a stain for compact myelin, was significantly decreased in the CC of $G p r 56^{-/-}$mice, compared with controls (Fig. 1a,b), suggestive of hypomyelination. Similarly, immunohistochemistry (IHC) and western blot analysis of myelin basic protein (MBP) and proteolipid protein (PLP), two markers for mature OLs and CNS myelin, showed significant reductions of the two proteins in $G$ pr $56^{-I-}$ mice compared with controls (Fig. 1c-e and Supplementary Fig. 3).

Gpr56 mutants possess fewer myelinated axons. To further assess CNS myelination phenotype in Gpr56-knockout mice, we performed transmission electron microscopy (TEM) analysis on cross-sections of the CC and the optic nerves of P28 Gpr56 ${ }^{+1-}$ and $G p r 56^{-1-}$ mice (Fig. 2a,b). Strikingly, there were significantly fewer myelinated axons in both the CC and optic nerves of Gpr56 $6^{-1-}$ mice compared with the controls (Fig. 2c,d). To evaluate whether axons of a certain caliber were more severely affected in the absence of GPR56, we quantified the relative frequency of myelinated axons with respect to their corresponding diameters. We detected no statistical difference between the two groups (Fig. 2e,f), although we observed a shift towards a higher caliber of axons being myelinated in the CC of Gpr56 ${ }^{-1-}$ mutants (Fig. 2e). Despite the significant reduction in the percentage of myelinated axons in $G$ pr56 $6^{-/-}$mutants, g-ratio analysis revealed no difference in myelin sheath thickness between the two groups (Supplementary Fig. 4a,b). We also observed no difference in axon diameter and the total number of axons (myelinated and unmyelinated) in the CC and optic nerves between the two groups of animals (Supplementary Fig. 4c-f). Interestingly, we observed normal levels of myelination at 6 months of age in the optic nerve (Fig. 2g,h), indicating the myelination defect was gradually corrected by ongoing OL production. Together, these data demonstrate that Gpr56 mutants are hypomyelinated at early stages and that this phenotype is not due to gross axon defects.

GPR56 is expressed in the OL lineage. The observation that Gpr56 mutation causes a reduction in the percentage of myelinated axons but not in the total number of axons suggests that GPR56 could regulate OL development. To test this hypothesis, we performed GPR56 expression profiling in the OL lineage by a series of both in vivo and in vitro immunostaining for GPR56 and markers of various stages of OL differentiation. Sox 2 was used for a glial progenitor cell marker 25 , Olig2 for the OL lineage ${ }^{26-28}$, PDGFR $\alpha$ and NG2 for OPCs ${ }^{29}, \mathrm{O} 4$ antigen for immature OLs ${ }^{30}$ and $\mathrm{MBP}^{31}$ for mature myelinating OLs. In the CC of wild-type (wt) postnatal day (P) 5 mouse, GPR56 was detected in Sox ${ }^{+}, \mathrm{Olig}^{+}{ }^{+}, \mathrm{NG}_{2}{ }^{+}$and $\mathrm{O}^{+}{ }^{+}$cells (Fig. $3 \mathrm{a}-\mathrm{d}, \mathrm{f}-\mathrm{i}$ ). By P10, the cells had matured into myelinating $\mathrm{MBP}^{+}$OLs, GPR56 could no longer be detected (Fig. 3e,j). To further verify this temporal expression profile and to perform quantitative evaluation of GPR56 expression at various stages of OL differentiation, we performed double immunostaining on wt OPCs, immature and mature OLs (Fig. 3k-v and Supplementary Fig. 5). GPR56 was detected in $\sim 80 \%$ of Olig ${ }^{+}$and $\sim 90 \% \mathrm{PDGFR}^{+}$cells (Fig. 3k-p,w). The percentage of GPR56 ${ }^{+}$cells steadily decreased in $\mathrm{O}_{4}{ }^{+}$immature and $\mathrm{MBP}^{+}$mature OLs (Fig. 3q-w). Taken together, our results indicate that GPR56 is expressed in glial progenitors and most OPCs and that this expression is downregulated in mature myelinating OLs, consistent with recent RNA-sequencing transcriptome data ${ }^{32}$. These results further support the notion that GPR56 could regulate OL development. Furthermore, our data showed $\sim 10 \%$ PDGFR $\alpha^{+}$ OPCs do not express GPR56, suggesting a heterogeneous nature of OPC population. This expression profile also explains the correction of myelination defects in mutants at 6 months of age (Fig. 2g,h).

Loss of Gpr56 results in fewer mature OLs in the CC. To test the hypothesis that GPR56 regulates OL development, we 
a
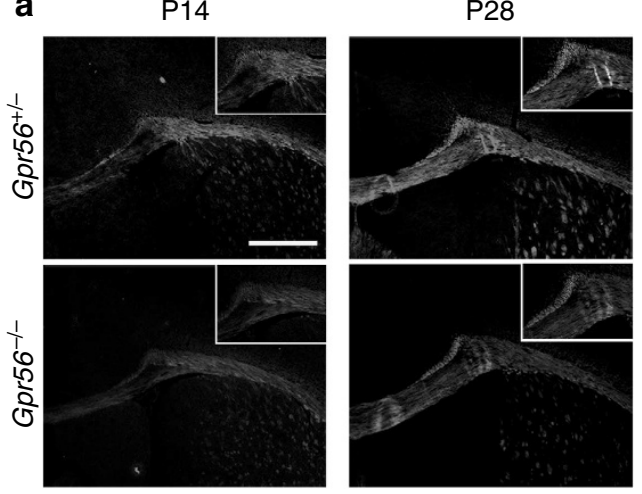

$\mathbf{C}$
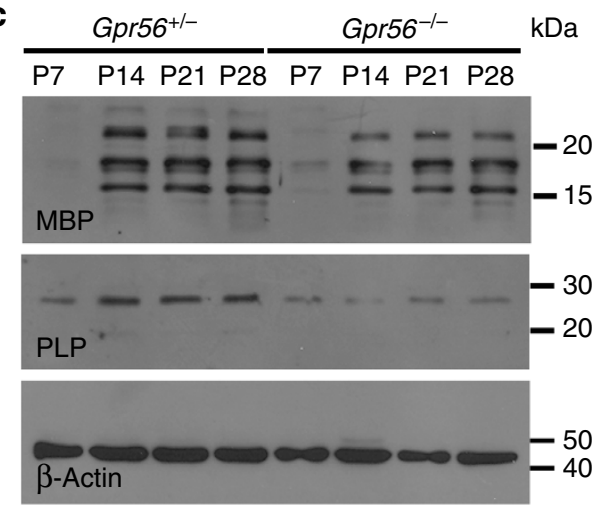

b
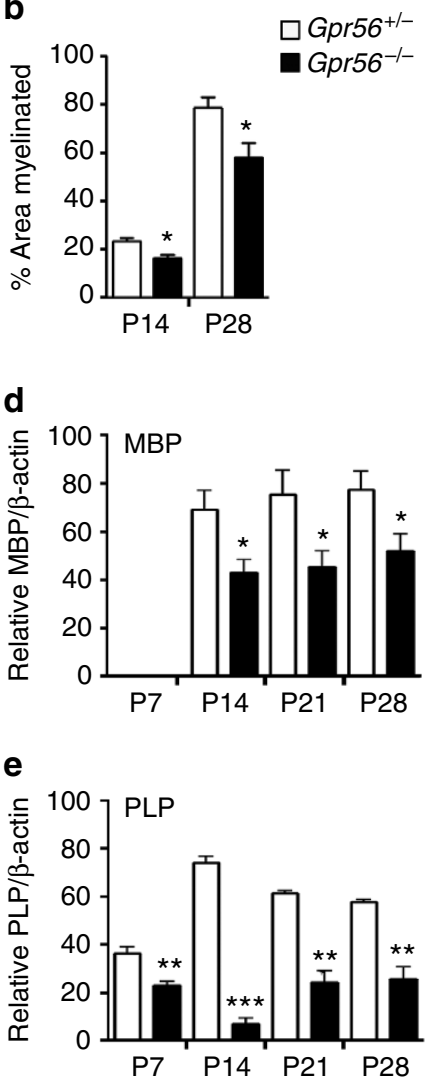

Figure 1 | Loss of GPR56 causes CNS hypomyelination in mice. (a) Reduced FluoroMyelin staining was observed in the CC of P14 and P28 brains of Gpr56 ${ }^{-/}$- mice (lower panel) compared with their littermate control (upper panel). Scale bar, $500 \mu \mathrm{m}$. (b) Bar graphs depicted percentage of area myelinated. Myelin is reduced at P14 ( ${ }^{\star} P=0.0144$; unpaired $t$-test, $n=4$ per genotype) and $\mathrm{P} 28\left({ }^{\star} P=0.0499\right.$; unpaired $t$-test, $n=3$ per genotype) in the CC of $\mathrm{Gpr56}^{-/-}$compared with controls. (c) Western blot analyses of MBP and PLP expression in the CC of $\mathrm{Gpr} 56^{+/-}$and $\mathrm{Gpr} 56^{-/-}$mice at P7-28. Loading control: $\beta$-actin. (d,e) Bar graphs depicted relative optical density of MBP and PLP to the loading control $\beta$-actin. MBP protein was significantly reduced in the CC of Gpr56 $6^{-/-}$compared with Gpr56 $6^{+/-}$littermates on P14 $\left({ }^{\star} P=0.0285\right), P 21\left({ }^{\star} P=0.0315\right)$ and P28 $\left({ }^{\star} P=0.0443\right)$. Unpaired $t$-test, $n=3$ per genotype. PLP protein was significantly decreased in the CC of Gpr56 ${ }^{-/}$- compared with Gpr56 ${ }^{+/-}$littermates on P7 $\left.\left({ }^{\star \star} P=0.0091\right), \mathrm{P} 14\left({ }^{\star \star \star} P<0.0001\right), \mathrm{P} 21{ }^{\star \star} P=0.0011\right)$ and P28 $\left.{ }^{\star \star} P=0.003\right)$. Unpaired $t$-test, $n=4$ per genotype. Error bars are means \pm s.e.m.

performed quantitative analysis of mature OLs in Gpr56-knockouts. We crossed Plp:eGFP transgenic reporter mice ${ }^{33}$ with Gpr56 $6^{-1-}$ mice to generate Plp:eGFP /Gpr56 $6^{+1-}$. Subsequent crossing with the F1 mice generated Plp:eGFP / Gpr56 $^{+1+}$, Plp:eGFP/Gpr56 ${ }^{+/-}$and Plp:eGFP /Gpr56 ${ }^{-1-}$ mice. In these mice, enhanced green fluorescent protein, driven by the $P l p$ promoter, mostly labels mature OLs ${ }^{34}$. At P7, we did not observe any difference between the number of EGFP ${ }^{+}$OLs in the CC of mice of Plp:eGFP /Gpr56 $6^{+1-}$ and Plp:eGFP /Gpr56 ${ }^{-1}$ - . In contrast, we observed significantly fewer EGFP ${ }^{+}$OLs in the CC of Gpr56 $6^{-1-}$ mice, compared with the controls, starting at P14 and continuing to P56 (Fig. 4a,b). Next, we quantified the total number of OPCs by performing Pdgfr $\alpha$ in situ hybridization (ISH) on P7 and P14 Gpr56 ${ }^{-/-}$mice and their littermate controls. We observed a significant reduction in the number of Pdgfra ${ }^{+}$cells in the CC of Gpr56 ${ }^{-/}$mice compared with the controls at both developmental stages analysed (Fig. 4c,d). To evaluate the status of immature OLs in the CC of Gpr56knockout mice, we measured levels of $2^{\prime}, 3^{\prime}$-cyclic nucleotide $3^{\prime}$-phosphodihydrolase (CNP), a marker for immature OLs ${ }^{1,26}$. We observed reduced CNP protein levels in the CC of $G$ pr56 ${ }^{-1-}$ mice, compared with their littermate controls (Supplementary Fig. 6), indicative of fewer $\mathrm{CNP}^{+}$immature OLs. Together, these data demonstrate that GPR56 is required for the proper development of OLs.
GPR56 is required for OPC proliferation. GPR56 was recently shown to regulate neural stem cell proliferation in the developing neocortex ${ }^{21}$. To test whether the reduced number of OPCs and mature OLs observed in Gpr56 knockouts is due to decreased OPC proliferation, we performed double immunostaining of NG2 and Ki67, markers for OPCs and proliferating cells, respectively, on postnatal brains of $G$ pr56 $6^{-I-}$ and their littermate controls. We chose to examine P14 brains based on our observation that fewer mature OLs were first observed at this stage (Fig. 4). A significantly reduced number of dual-positive cells was detected in $G$ pr $56^{-1-}$ brains, compared with the controls (Fig. 5a,b), suggesting that GPR56 has a role in regulating OPC proliferation, either directly or indirectly.

Next, we carried out cell proliferation assays in vitro using OPCs isolated from P5 brains of Gpr56 $6^{-/}$mice and their littermate controls by immunopanning ${ }^{35-37}$. After culturing for 4 days in the presence of PDGF-AA, a major OPC mitogen, we found that the percentage of dividing OPCs, represented by $\mathrm{Ki}^{+} 7^{+}$in total $\mathrm{PDGFR} \alpha^{+}$population, was significantly reduced in OPCs derived from Gpr56 $6^{-1-}$ mouse brains compared with controls (Fig. 5c,d).

In light of the expression pattern of GPR56 in OL development and the reduced OPC proliferation in the absence of GPR56, we hypothesized that GPR56 is required for OPCs to remain in cell cycle. To test this hypothesis, we performed cell cycle exit assay 
a

Corpus callosum-P28
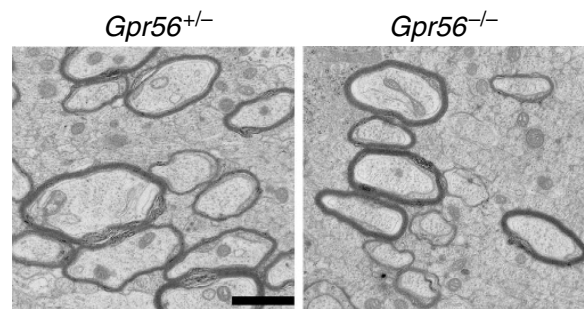

C

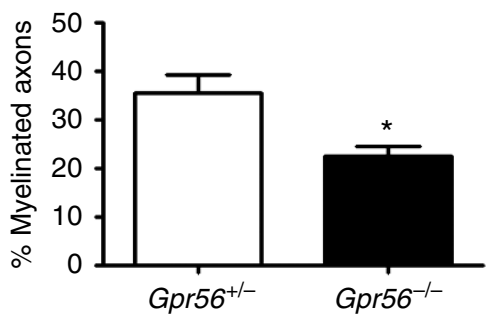

e

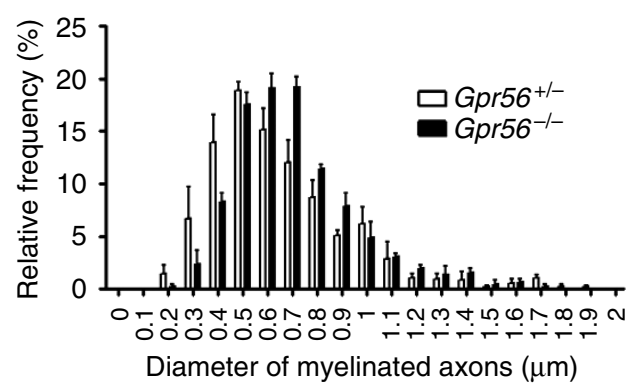

g

$\mathrm{Gpr56}^{+/-}$

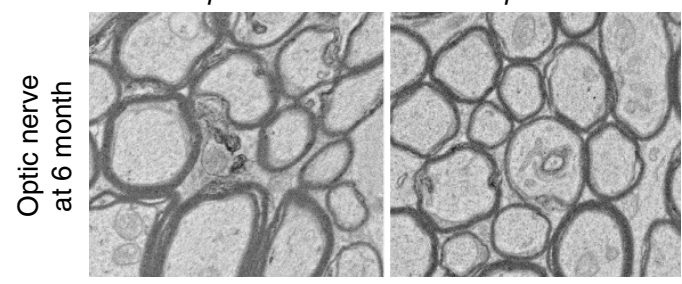

b

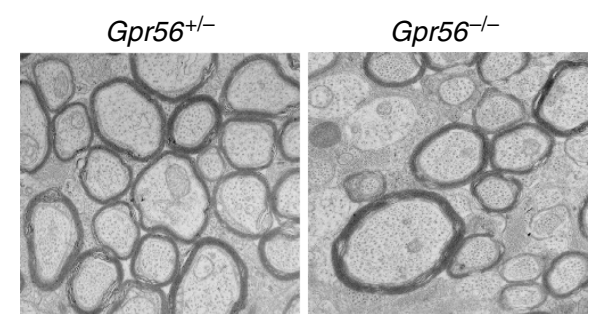

d

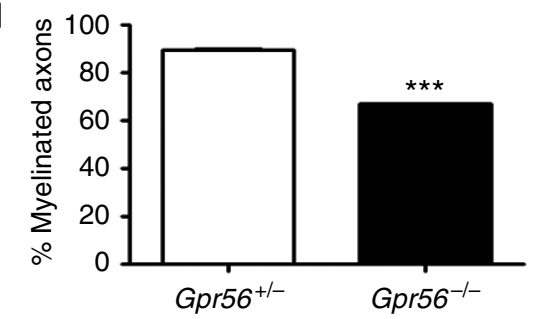

f
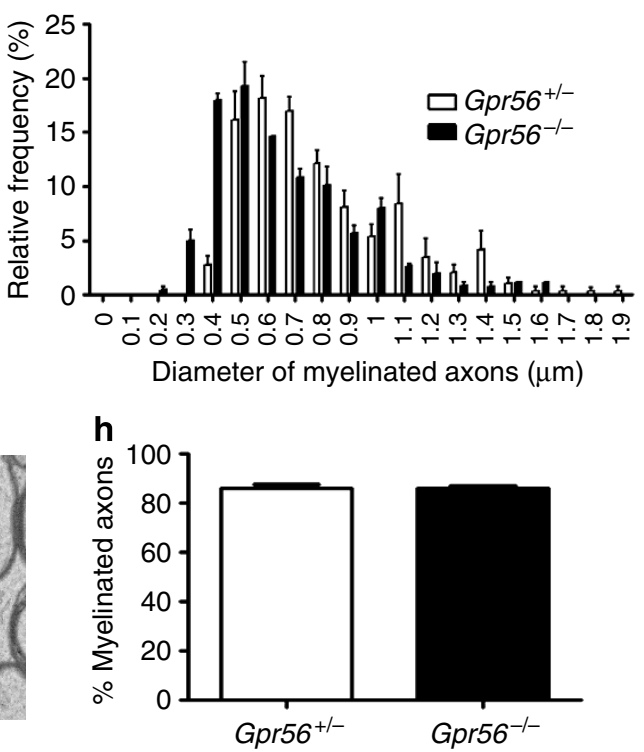

Figure 2 | Loss of GPR56 results in fewer myelinated axons in the CC and optic nerves at P28. (a,b) Representative TEM images from P28 CC (a) and P28 optic nerves (b) of Gpr56 $6^{+/-}$(left) and Gpr56 ${ }^{-/-}$(right) mice. (c,d) Percentages of myelinated axons were quantified in the CC (c) and optic nerves (d) of Gpr56 $6^{-/-}$mice $\left({ }^{\star} P=0.0264\right.$ (c); ${ }^{\star \star \star} P=0.0004$ (d); paired t-test, $n=3$ per genotype). (e,f) The distribution of myelinated axons with respect to the axon diameter was comparable in the CC (e) and optic nerve (f) between Gpr56 $6^{+/-}$and $G p r 56^{-/-}$mice $(P=0.3185$ (e); $P=0.321$ (f); paired t-test, $\mathrm{n}=3$ per genotype). (g) Representative TEM images from the optic nerves at 6 months of $\mathrm{Gpr} 56^{+/}-($left) and $\mathrm{Gpr}^{-1-}$ (right) mice. (h) Percentage of myelinated axons was quantified ( $P=0.7680$; paired $t$-test, $n=3$ per genotype). Error bars are means \pm s.e.m. Scale bar, $1 \mu \mathrm{m}$.

by pulsing Gpr56 $6^{-1-}$ pups and their wt littermate controls with BrdU on P13, $24 \mathrm{~h}$ before brain harvesting, followed by double IHC of BrdU and Ki67. BrdU and Ki67 dual-positive cells represent those cells that remain in the cell cycle, whereas $\mathrm{BrdU}^{+} ; \mathrm{Ki}^{-} 7^{-}$cells represent those that have already exited the cell cycle at the time of analysis. We found significantly fewer $\mathrm{BrdU}^{+} ; \mathrm{Ki} 7^{+}$double-positive cells (Fig. 5e,f) as well as a lower percentage of $\mathrm{Ki} 67^{+}$cells relative to the total $\mathrm{BrdU}^{+}$population (Fig. $5 \mathrm{~g}$ ) in the CC of $G$ pr56 $6^{-/-}$mice in contrast to their littermate controls. Cyclin-dependent kinase 2 (CDK2) controls OPC cell cycle progression ${ }^{38,39}$. To further demonstrate that GPR56 keeps OPCs in cell cycle, we performed western blot analysis of CDK2 in acutely isolated OPCs from P6 Gpr56-1mice and their littermate controls by immunopanning. We observed a significant reduction of CDK2 protein in the OPCs isolated from $G$ pr56 $6^{-1-}$ mice compared with their littermate controls (Fig. 5h,i), supporting that OPCs prematurely exit the cell cycle in the absence of GPR56.

GPR56 promotes OPC proliferation via the RhoA pathway. Two signalling pathways have been reported to be downstream of GPR56 activation, depending on the cell type. In neural progenitor cells, GPR56 signals via the RhoA pathway ${ }^{40}$, whereas it activates protein kinase $\mathrm{C} \alpha(\mathrm{PKC} \alpha)$ in melanoma cells ${ }^{41}$. To investigate the signalling mechanism of GPR56 in the developing white matter, we performed active RhoA pull down assays and PKC $\alpha$ western blot analyses, using P7 optic nerves of $G p r 56^{-1-}$ mice and their littermate controls. Whereas the level of PKC $\alpha$ was not affected by deleting Gpr56 (Supplementary Fig. 7a,b), we observed significantly lower levels of active RhoA in the optic nerves of $G$ pr56 $6^{-1-}$ mice compared with their littermate 


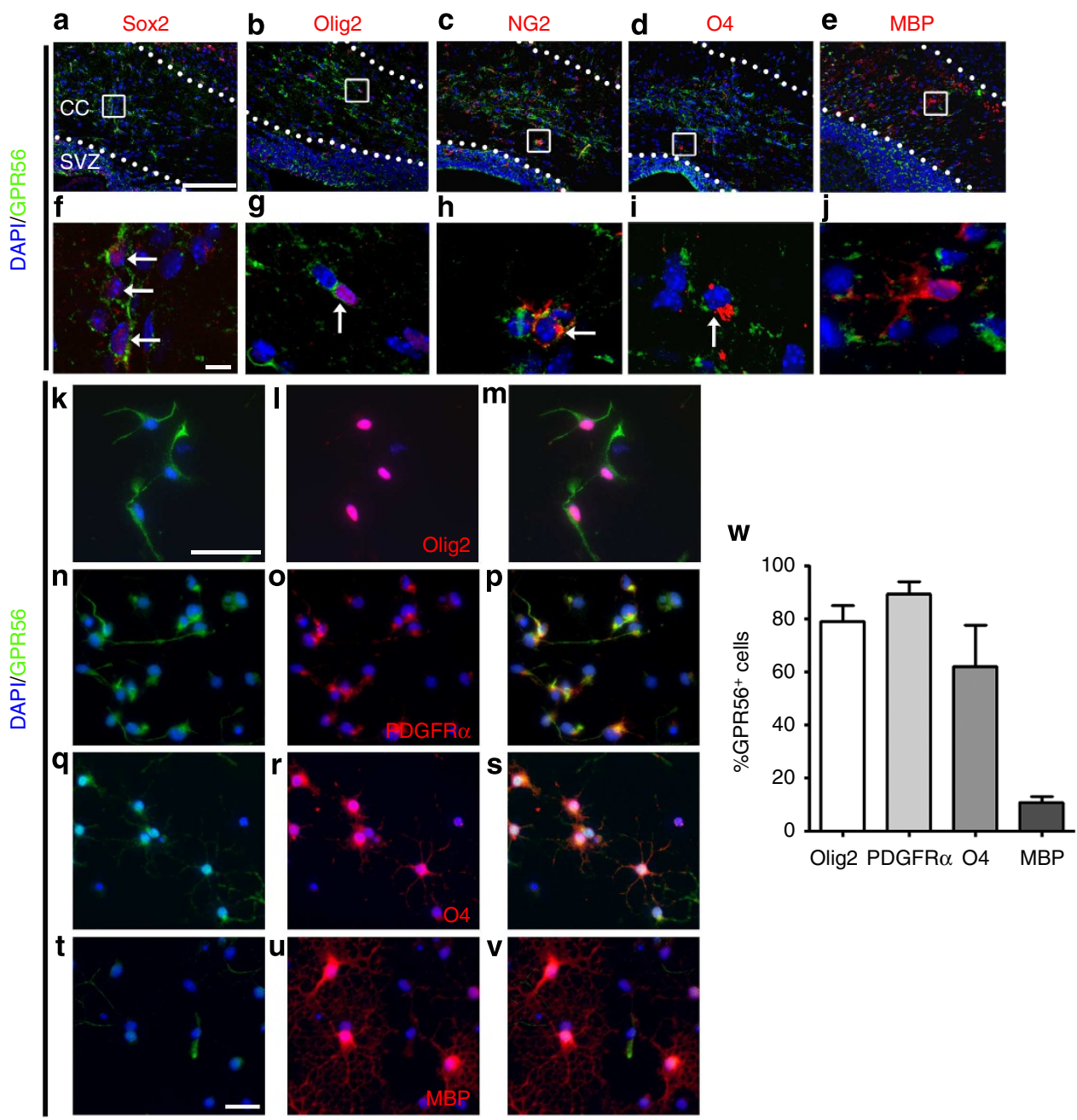

Figure 3 | GPR56 is expressed in the OL lineage. (a-e) Double IHC for GPR56 (green) and Sox2, Olig2, NG2 and O4 (red) on wt P5 brains as well as MBP (red) on wt P10 brains. (f-j) Higher magnification of the boxed area in a-e. Scale bar, a-e, $100 \mu \mathrm{m} ; \mathbf{f}-\mathbf{j}$, $10 \mu \mathrm{m}$. (k-v) Double immunocytochemistry of GPR56 (green) and various markers (red) on cultured primary OPCs and OLs that were either cultured for 2-4 days in proliferating media (k-p) or 3 days in differentiation media $(\mathbf{q}-\mathbf{v})$. Scale bar, $50 \mu \mathrm{m}$. (w) Bar graph depicts percentage of OL lineage cells expressing GPR56. CC, corpus callosum; $\mathrm{SVZ}$, subventricular zone. Error bars are means \pm s.e.m.

controls (Fig. 5j,k). These data suggest that GPR56 signals through RhoA to regulate OPC proliferation.

GPR56 has no effect on OPC survival and maturation. Another potential cause for reduced mature OLs in Gpr56 mutants is increased OPC cell death in the absence of Gpr56. Cell death assays revealed comparable numbers of TUNEL-positive apoptotic cells in the subventricular zone and corpus callosum (CC) in both control and Gpr56 $6^{-1-}$ brains at P14 (Fig. 6a,b). Furthermore, judging by the similar distribution pattern of Pdgfra ${ }^{+}$cells at P14 (Fig. 4c) as well as EGFP ${ }^{+}$cells from P7 to P56 (Fig. 4a), in the CC of Gpr56 ${ }^{+/-}$and $G p r 56^{-/-}$mice, GPR56 likely has no influence on OPC migration.

In addition, we suspected that GPR56 does not affect the elaboration of branched processes of OLs based on the fact that GPR56 is downregulated in terminally differentiated $\mathrm{MBP}^{+}$OLs (Fig. 3) and that deleting Gpr56 has no effect on the myelin thickness (Supplementary Fig. 4a,b). To confirm this notion, we cultured OPCs isolated from $G$ pr56 ${ }^{-/-}$and their littermate controls on either poly-D-lysine (PDL) or laminin-coated coverslips for 7 days in the presence of thyroid hormone to induce terminal differentiation. Indeed, we did not observe any difference in the myelin sheath area between $G p r 56^{+1-}$ and Gpr56-I- OLs cultured on laminin or PDL (Fig. 6c,d). Moreover, deleting Gpr56 has no effect on the ability of OPCs to terminally differentiate (Fig. 6e,f).

GPR56 functions autonomously in the OL lineage. To define the cell autonomy of GPR56 during OL development, we generated a new targeted allele of Gpr56 containing loxP sites flanking exons 4-6, hereafter referred to as the floxed $(f l)$ allele of Gpr56 (Fig. 7a,b). On crossing with tissue-specific Cre transgenic mice, exons 4-6 are deleted, causing a frameshift leading to a deletion of all splicing variants of Gpr56. We first crossed Gpr56 $6^{\text {llfl }}$ mice with EIIA-Cre mice, a universal Cre-line ${ }^{42}$, to create a constitutive knockout mouse line. Western blot analysis failed to detect any GPR56 protein in the brains of Gpr56 $6^{f l / f l}$;EIIA-cre ${ }^{+/-}$mice (Fig. 7c), confirming the efficacy of our targeting strategy. We began our analysis using Pdgfro-CreERT transgenic mice ${ }^{43,44}$ to excise Gpr56 in OPCs by daily administration of tamoxifen from P10 to P14. We chose P10-14 based on the observations that oligodendrogenesis starts perinatally and peaks at P14 (refs 45-48). We performed $P l p$ ISH on P21 CC of Gpr56 $6^{f l f l} ; P d g f r \alpha-c r e^{+/-}$and their 
a
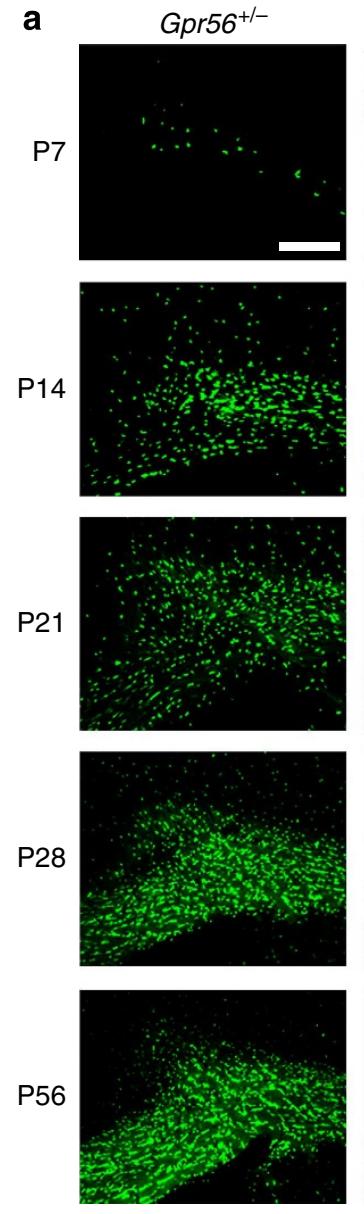

$\mathrm{Gpr56}^{-/-}$
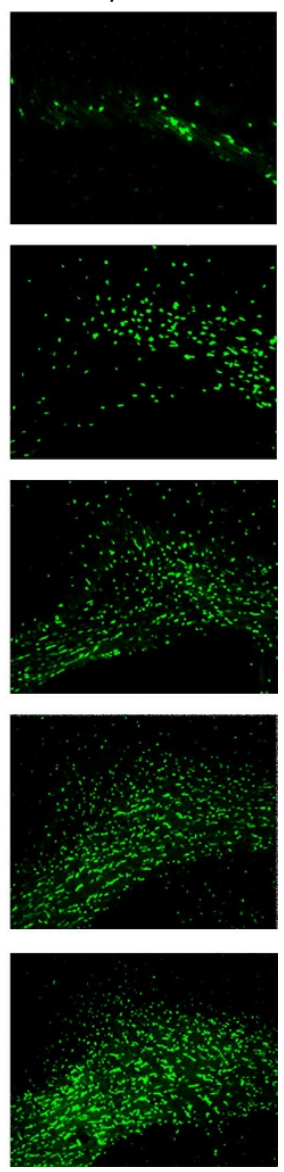

b

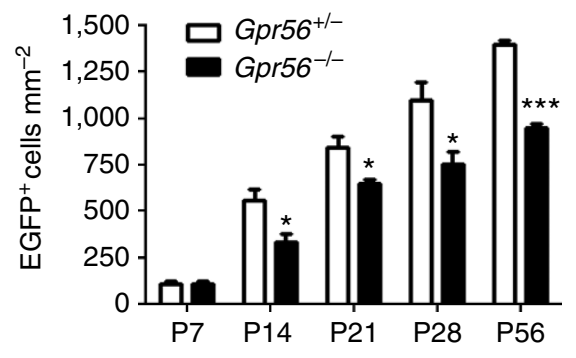

c
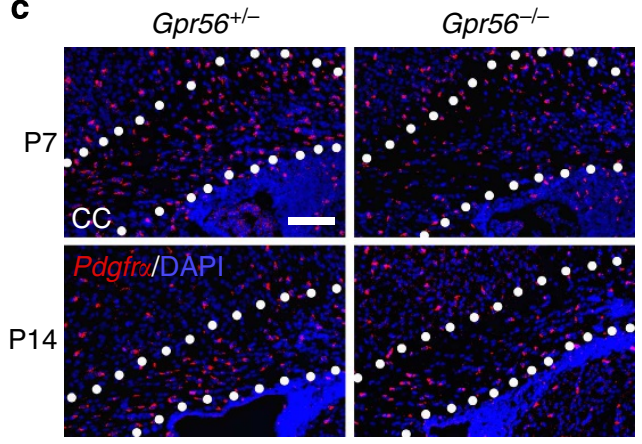

d

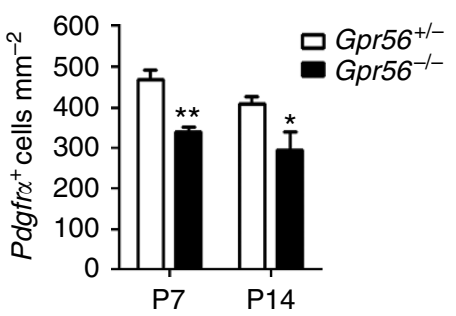

Figure 4 | Loss of GPR56 results in fewer mature OLs and OPCs in the CC. (a) Representative images of EGFP + (green) mature OLs in the CC of Gpr56 ${ }^{+/-}$(left panel) and Gpr56-/- (right panel) mouse brains from P7 to P56. Scale bar, $200 \mu \mathrm{m}$. (b) Quantification of EGFP + cells in the CC. P7 $(P=0.9686), \mathrm{P} 14\left({ }^{\star} P=0.0373\right), \mathrm{P} 21\left({ }^{\star} P=0.0314\right), \mathrm{P} 28\left({ }^{\star} P=0.0478\right)$ and $\mathrm{P} 56\left({ }^{\star \star \star} P<0.0001\right)$, unpaired $t$-test, $n=3$ per genotype. (c) Representative images of ISH of Pdgfra (red). Scale bar, $100 \mu \mathrm{m}$. (d) Quantification of Pdgfr ${ }^{+}$cells in the CC. P7 ( $\left.{ }^{\star \star} P=0.0033\right), P 14\left({ }^{\star} P=0.0327\right)$, unpaired $t$-test, $n=4$ per genotype. Error bars are means \pm s.e.m.

littermate controls (Fig. 7d). A significantly fewer number of $\mathrm{Plp}^{+}$mature OLs was found in the CC of Gpr56 flff; Pdgfr $\alpha-\mathrm{cre}^{+1-}$, compared with the controls (Fig. 7e), demonstrating that GPR56 regulates OL development in a cell-autonomous manner.

\section{Discussion}

Whereas a clear role has been established for GPR56 in cerebral cortical development ${ }^{12,19,40,49,50}$, nothing was known about its function in CNS myelination. We demonstrate here that GPR56 is a novel regulator of OL development. Consistent with a previous report that GPR56 promotes neural stem cell proliferation in the developing neocortex ${ }^{21}$, we show that developing OPCs require GPR56 to remain in a proliferating state by demonstrating that (1) GRP56 is expressed robustly in Sox $2^{+}, \mathrm{NG}_{2}{ }^{+}$and PDGFR $\alpha^{+}$cells, diminished in $\mathrm{O} 4{ }^{+}$cells and strongly downregulated in $\mathrm{MBP}^{+}$OLs. (2) There are significantly fewer $\mathrm{NG}^{+}{ }^{+} ; \mathrm{Ki}_{6}{ }^{+}$double-positive cells in the CC of Gpr56 knockouts. (3) Fewer PDGFR $\alpha^{+}$OPCs derived from Gpr56 knockouts proliferate following 4 days of culture in vitro. (4) Loss of GPR56 causes OPCs to exit cell cycle prematurely. (5) Conditionally ablating Gpr56 in OPCs results in a significantly reduced number of mature OLs. The fact that deleting Gpr56 has no effect on axon diameter or the total number of axons further supports that GPR56 has an autonomous function in OPC development.

ECM and ECM receptor loss-of-function studies in mice have demonstrated that cell-matrix interactions are important for gliogenesis and myelination ${ }^{3}$. For example, many studies have shown that laminin-integrin interactions regulate OL process dynamics $^{3}$ and $d y / d y$ mutants ( $\alpha 2$ laminin hypomorphs) have regional defects in CNS myelination as well as delayed OL maturation $^{4,5}$. These published studies are consistent with the phenotypes that we observed in $G p r 56^{-1-}$ mutants. Moreover, aGPCRs often bind ECM proteins and we hypothesize that GPR56-ECM interactions regulate OL development. It is unclear which ECM ligand activates GPR56 in the developing white matter. Collagen III is the ligand of GPR56 in the developing cerebral cortex. However, collagen III is mainly expressed in the meninges and blood vessels ${ }^{40}$, making it an unlikely ligand of GPR56 during OPC development.

GPR56 has a very long and poorly characterized N-terminal fragment, allowing for the possibility of multiple binding partners. Indeed, GPR56 also binds to tissue transglutaminase (TG2) in melanoma cells ${ }^{51}$. TG2 is an inducible transamidating acyltransferase that has several distinct biochemical functions ${ }^{52-54}$. Interestingly, the enzymatic activity of TG2 is low in the early embryonic mouse brain but increases through development, peaks on the day of birth (P0) and is maintained at high levels 
a
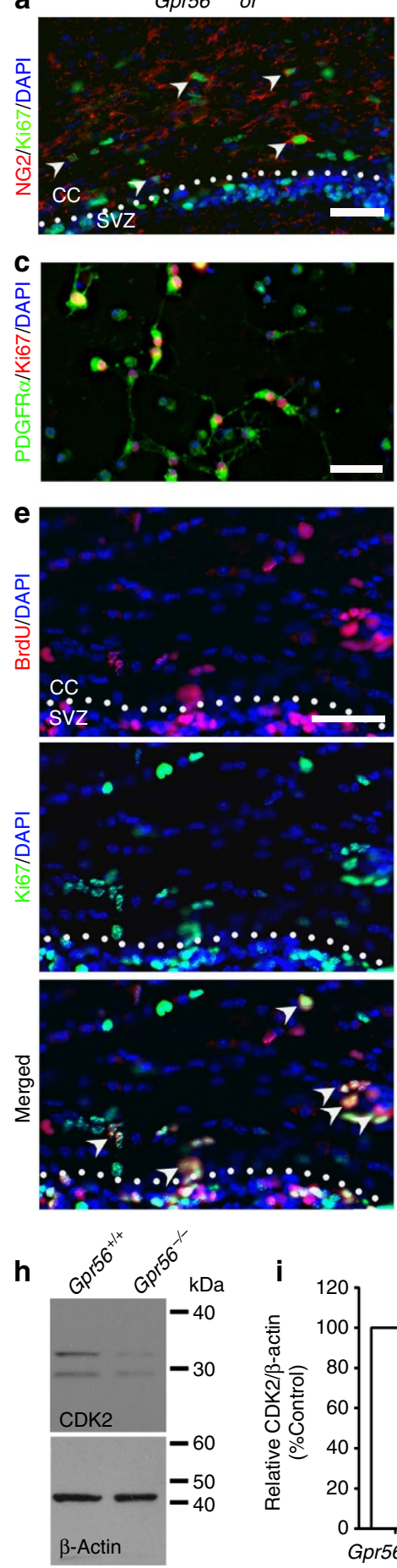
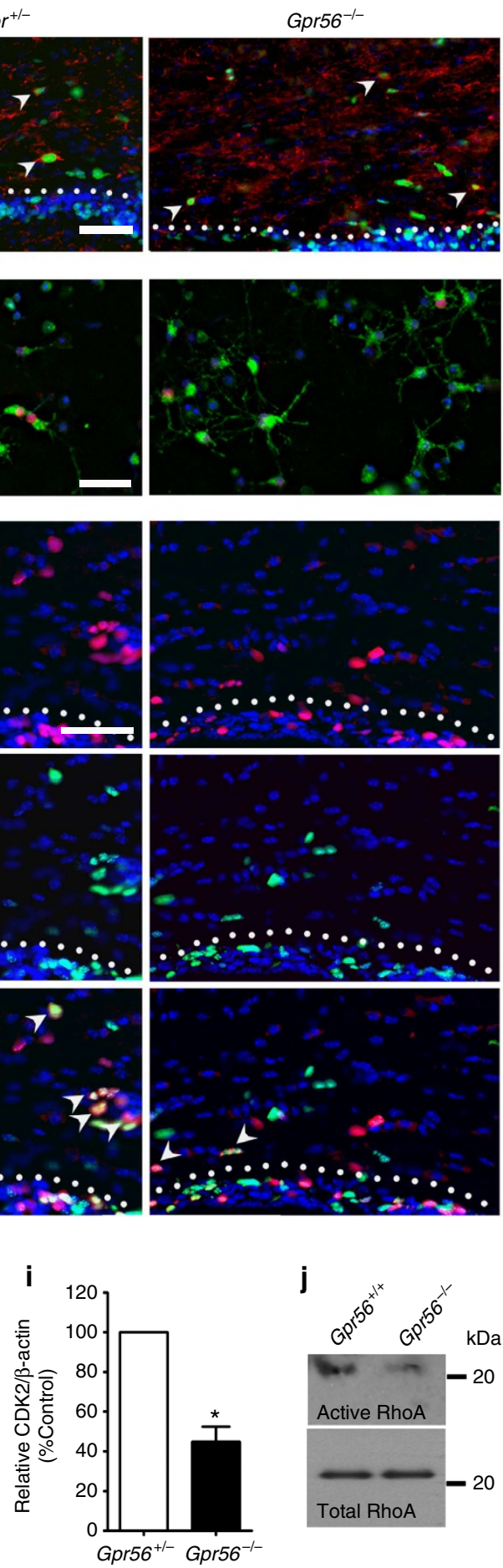
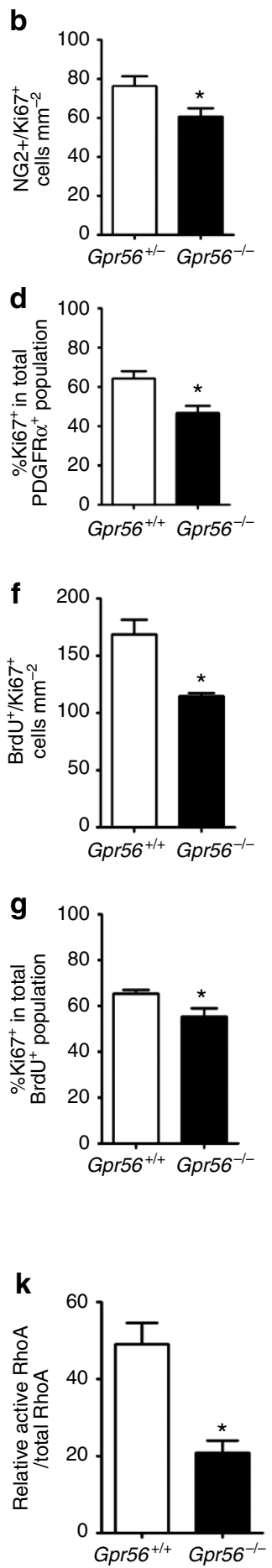

Figure 5 | Loss of GPR56 leads to fewer proliferating OPCs. (a) Representative images of NG2 (red) and Ki67 (green) double IHC in the CC of Gpr56 + / and Gpr56-/ - P14 mice. Arrowheads indicate double-positive cells. (b) Quantification of NG2 and Ki67 dual-positive cells. The asterisks represent significance based on unpaired $t$-test. $P=0.0382 ; n=6$ per genotype. (c) Representative images of PDGFR $\alpha$ (green) and Ki67 (red) double immunostaining on OPCs after cultured for 4 days in proliferation media. (d) Quantification of PDGFR $\alpha$ and Ki67 dual-positive OPCs. The asterisks represent significance based on paired $t$-test. $P=0.0156 ; n=3$ per genotype. (e) Representative images of BrdU (red) and Ki67 (green) double staining on P14 Gpr56 $+/+$ and $\mathrm{Gpr}^{-1} 6^{-/}$brains that were pulsed with BrdU $24 \mathrm{~h}$ before. Arrowheads indicate double-positive cells. (f) The number of BrdU and Ki67 double-positive cells was quantified in the CC of Gpr56 $6^{-1}$ mice compared with controls. The asterisks represent significance based on unpaired $t$-test. $P=0.0153 ; n=3$ per

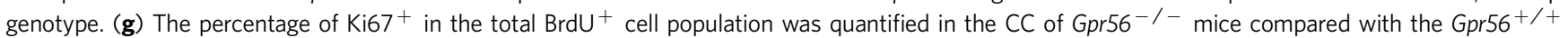
controls. The asterisks represent significance based on unpaired $t$-test. $P=0.0289 ; n=4$ per genotype. (h) Western blot depicting CDK2 protein level in actually isolated OPCs from Gpr56 $6^{+/+}$and $\mathrm{Gpr} 56^{-/-} \mathrm{P} 6$ mice. (i) The relative CDK2 protein levels were shown. The asterisks represent significance based on paired $t$-test. $P=0.0179 ; n=3$ per genotype. (j) Western blot of active RhoA (top panel) and total RhoA (bottom panel) in the optic nerves of $G p r 56^{+/+}$ and $G$ pr56 $6^{-/-}$mice. (k) The relative level of active RhoA to total RhoA was presented. The asterisks represent significance based on unpaired $t$-test. $P=0.0122 ; n=3$ per genotype. CC, corpus callosum; SVZ, subventricular zone. Scale bar, $50 \mu \mathrm{m}$. Error bars are means \pm s.e.m. 
a

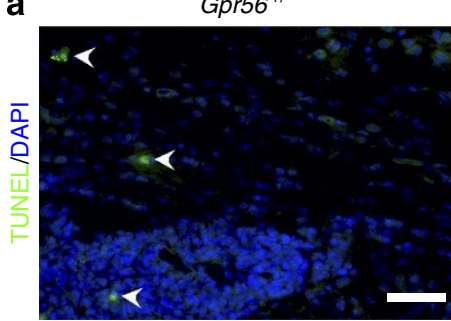

C
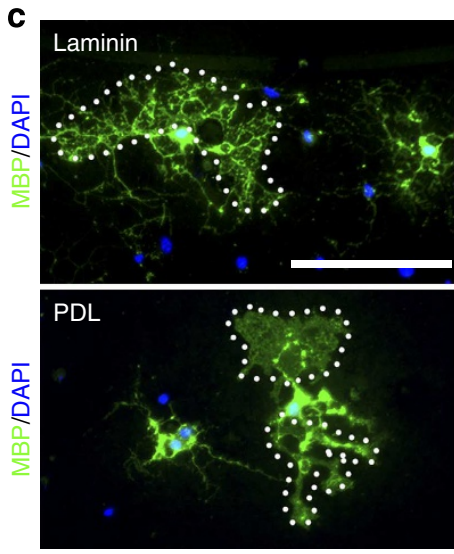

e

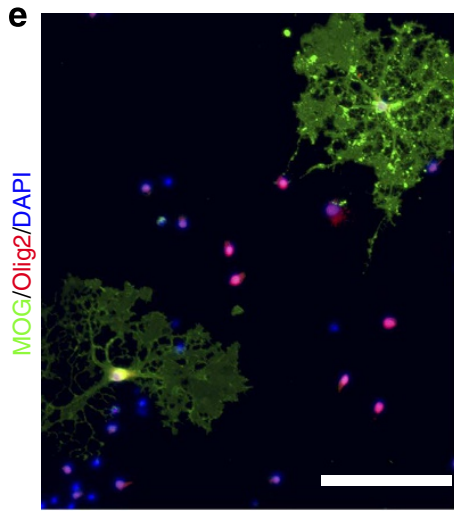

Gpr56 ${ }^{-/-}$
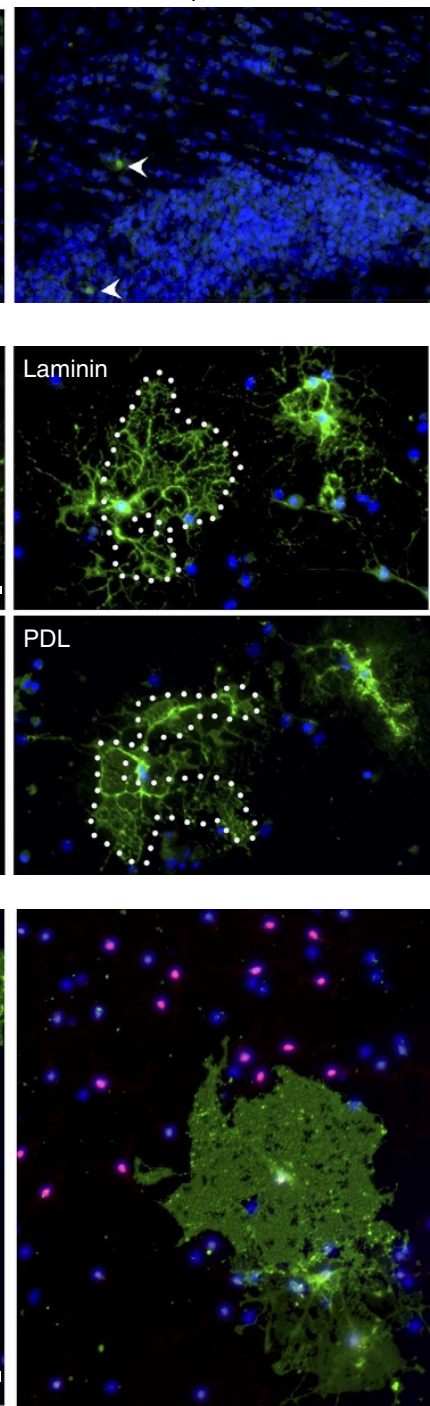

b

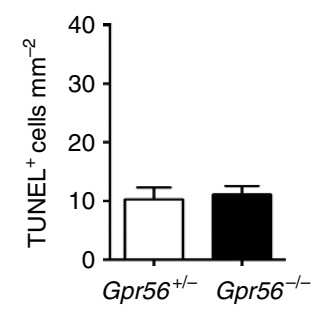

d

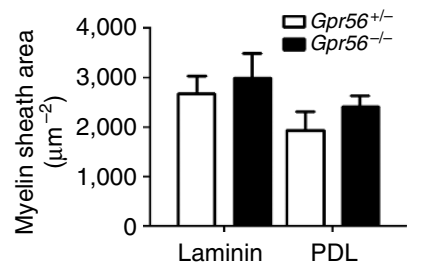

f

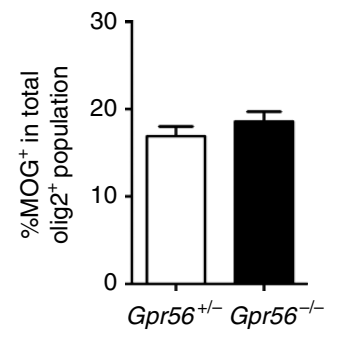

Figure 6 | Loss of GPR56 has no effect on OPC survival, OL process elaboration and maturation. (a) Representative images of TUNEL ${ }^{+}$cells (green; arrowheads) in the SVZ and CC of Gpr56+/- and Gpr56 ${ }^{-/-}$mice at P14. Scale bar, $50 \mu \mathrm{m}$. (b) Quantification of TUNEL cells. P=0.7573; unpaired $t$-test, $n=6$ per genotype. (c) Representative images of $\mathrm{MBP}^{+}$(green) OLs cultured for 7 days on either laminin (upper panel) or poly-D-lysine (PDL, lower panel) in differentiation media. Scale bar, $100 \mu \mathrm{m}$. (d) Quantification of myelin sheath area of OLs cultured on laminin $(P=0.2043)$ or PDL $(P=0.3027)$ (paired $t$-test, $n=3$ per genotype). (e) Representative images of MOG (green) and Olig2 (red) double labelling on OLs derived from Gpr56 $+/-$ and Gpr56 ${ }^{-/-}$mice cultured for 7 days in differentiation medium. Scale bar, $100 \mu \mathrm{m}$. (f) Percentage of differentiated OLs (MOG ${ }^{+}$) in total OLs $\left(\mathrm{Olig2}^{+}\right.$) was quantified. $P=0.5044$; paired $t$-test, $n=3$ per genotype. Error bars are means \pm s.e.m.

up to P56 (ref. 55), consistent with a potential role in OL development and myelination. TG2 activity has recently been implicated in CNS remyelination, as $\mathrm{Tg} 2^{-}$- mice remyelinate more slowly than wt animals in cuprizone models ${ }^{56}$. Moreover, OPC differentiation is inhibited in vitro on addition of TG2 inhibitors ${ }^{56}$. Therefore, it is possible that TG2 functions as the ligand of GPR56 during white matter development. Future studies are needed to test this hypothesis.

Further elucidation of GPR56 signalling in OPCs depends on the identification of its ligand. $\mathrm{G} \alpha_{12 / 13}$ and RhoA are the downstream effectors of GPR56 in neural progenitor cells and cultured cell lines ${ }^{40,57,58}$. On the basis of the fact that inactivation of RhoA is required for the terminal differentiation of OLs ${ }^{59-62}$ and that GPR56 ceases to be expressed in mature myelinating OLs, RhoA could signal downstream of GPR56 in the developing OPCs. Indeed, we detected significantly reduced level of active
RhoA in the optic nerves of Gpr56 $-/$ mice compared with their littermate controls.

In summary, we reveal a novel GPCR that regulates proper levels of CNS myelination by autonomously promoting OPC proliferation via the RhoA pathway (Fig. 8). Identification and characterization of signalling molecules involved in OL development may provide potential therapeutic targets for enhancing remyelination. Given the fact that GPCRs are the major targets for drug discovery ${ }^{63}$, the present study presents a potential new target for therapeutics to promote myelin repair in individuals afflicted with demyelination or dysmyelination.

\section{Methods}

Mice. All animals were treated according to the guidelines of the Animal Care and Use Committee at Boston Children's Hospital. The Gpr56-knockout mice were obtained from Genentech/Lexicon Genetics. The mutant mice were originally 
a

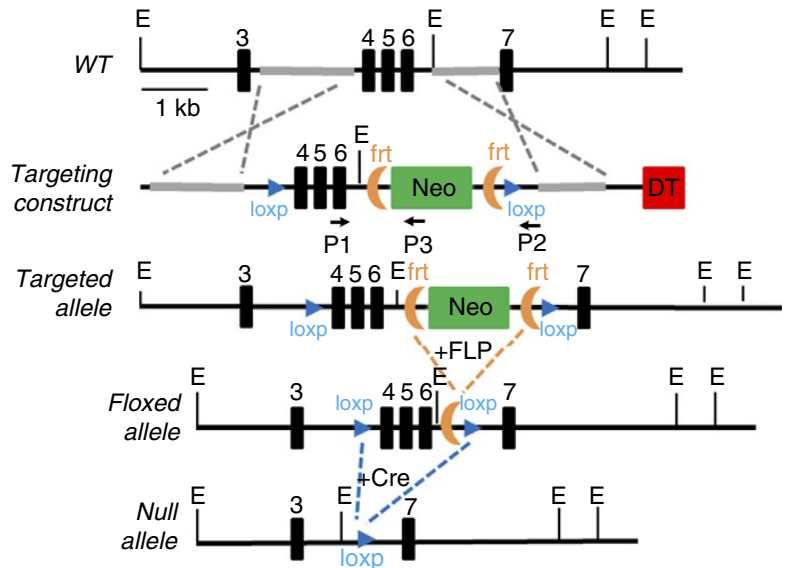

b

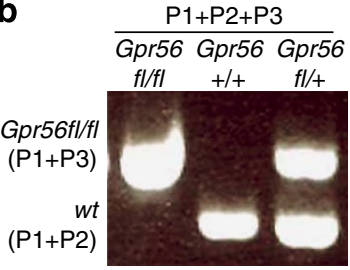

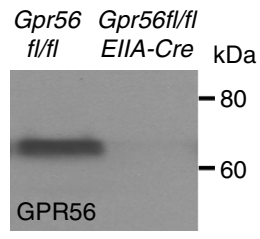

d

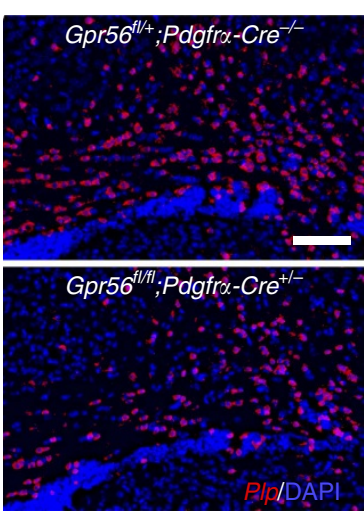

e

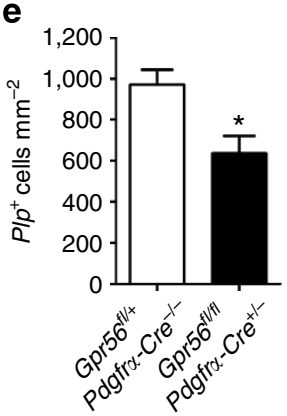

Figure 7 | OPC-specific deletion of Gpr56 leads to fewer mature oligodendrocytes. (a) Schematic drawing of targeting strategy. Exons 4, 5 and 6 were flanked with two loxP sites. (b) PCR genotyping revealed Gpr56 ${ }^{\mathrm{fl} / \mathrm{fl}}, \mathrm{Gpr} 56^{+/+}$and Gpr56 $6^{f / /}+$ alleles. (c) Western blot analysis shows absence of GPR56

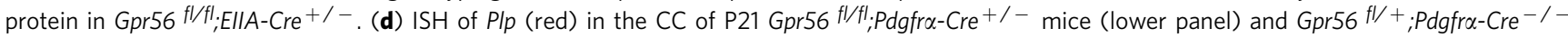
controls (upper panel) that received tamoxifen for five consecutive days during P10-P14. Scale bar, 100 $\mu$ m. (e) Quantification of Plp ${ }^{+}$mature OLs. The asterisks represent significance based on unpaired $t$-test. $P=0.0177$; unpaired $t$-test, $n=4$ per genotype. Error bars are means \pm s.e.m.

created in a 129/BL6 background, but were derived into the FvB strain and bred into $\mathrm{BALB} / \mathrm{c}$ strain resulting in a mixed genetic background of the mutant mice of $129 / \mathrm{BL} 6 / \mathrm{FvB} / \mathrm{BALB} / \mathrm{c}^{19}$. Genotyping was performed by PCR using the following primers: A (5'-CGAGAAGACTTCCGCTTCTG-3'), B (5'-AAAGTAG CTAAGATGCTCTCC-3') and Neo (5'-GCAGCGCATCGCCTTCTATC- $\left.3^{\prime}\right)$. Plp:eGFP transgenic mice ${ }^{33}$ were bred into the Gpr56 line to create Plp:eGFP/ Gpr56 $6^{+1+}$, Plp:eGFP/Gpr56 $6^{+1-}$ and Plp:eGFP/Gpr56 $6^{-1-}$ mice.

Gpr56 $6^{l /+}$ mice were generated at the Mouse Gene Manipulation Core at Boston Children's Hospital. The targeting vector (Fig. 7a) was introduced into C57BL/6 ES cells to generate targeted ES clones. Chimeric mice derived from targeted ES cell clone were crossed with C57BL/6 mice to obtain Gpr56 $6^{f l+}$ mice. We used the following primers to detect the presence of the floxed allele: primer 1: $5^{\prime}$-tggtagctaacctactccaggagc- $3^{\prime}$, primer 2: $5^{\prime}$-ggtgactttggtgttctgcacgac- $3^{\prime}$ and primer 3: $5^{\prime}$-cacgagactagtgagacgtgctac- $3^{\prime}$

$P d g f r \alpha-C r e / E R T$ mice in a C57BL/6 background were purchased from Jackson Laboratories (Cat\# 018280) and were crossed with Gpr56 fl/fl mice to generate Gpr56 ${ }^{l l f l} ;$ Pdgfra-Cre ${ }^{+1-}$ mice and their littermate controls. EIIA-Cre mice in a C57BL/6 background were purchased from Jackson Laboratories (Cat\# 003724) and were crossed with Gpr56 $6^{f / f l}$ mice to generate Gpr56 $6^{l / f l} ;$ :EIIA-Cre ${ }^{+l-}$ mice and controls. Only male mice were used for time points of P28 and later to avoid cyclic oestrogen effect on myelination, whereas both male and female mice were used for earlier time points.

Antibodies. The following primary antibodies were used for IHC or western blot analyses: mouse anti-GPR56 (H11) $(1: 200)^{40}$ and rabbit anti-GPR56 (199) $(1: 200)^{19}$, rabbit anti-MBP (Millipore; Cat \#AB980, 1:200), rat anti-MBP (Abcam, Cat\# ab7349), mouse anti-O4 (Millipore; Cat \#MAB345, 1:400), rabbit anti-NG2 (Millipore; Cat \#AB5320, 1:200), goat anti-Sox2 (Santa Cruz; Cat \#sc-17320, 1:400), rabbit anti-Olig2 (kind gift from Charles Stiles, 1:10,000), rat anti-PDGFR $\alpha$ (BD Bioscience; Cat \#558774, 1:500), rabbit anti-PDGFR $\alpha$ (Cell Signaling Technologies; Cat \#3164S, 1:500) and rat anti-Ki67 (Affymetrix eBioscience; Cat \#14-5698-80, 1:100), rat anti-BrdU (Accurate Chemical and Scientific Corporation; Cat \#OBT0030S, 1:100), rabbit anit-PLP (Abcam, Cat \#ab28486, 1:1,000), mouse antiRhoA (Cytoskeleton, Cat\# ARH03-A, 1:500), mouse anti-CDK2 (Santa Cruz; Cat \#sc-6248, 1:1,000), mouse anti- $\beta$-actin (Sigma, Cat \#A5044, 1:5,000) and mouse anti-Ki67 (BD Bioscience; Cat \#550609, 1:100). Secondary antibodies were goat anti-mouse or anti-rat conjugated with either Alexa 488 (Life Technologies,
1:1,000) or Alexa 546 (Life Technologies, 1:1,000) and goat anti-rabbit conjugated with Alexa 546 or 555 (Life Technologies, 1:1,000), goat anti mouse or rabbit IgGHRP (Sigma, Cat\# A4416 or A6154, 1:3,000).

Histology analyses. Mouse brains were harvested after perfusion, fixed with $4 \%$ PFA, cryoprotected with $30 \%$ sucrose and embedded in OCT. IHC was carried out as previous described ${ }^{64}$. In brief, after antigen retrieval in Retrievagen A Solution (BD Pharmingen), brain sections were washed with PBS, blocked with $10 \%$ goat serum, $1 \%$ bovine serum albumin (BSA) and $0.1 \%$ Triton X-100 in PBS for $1 \mathrm{~h}$ at room temperature before incubating with the primary antibody overnight at $4{ }^{\circ} \mathrm{C}$. Primary antibodies were visualized by incubating the sections with the appropriate fluorophore-conjugated secondary antibody for $1 \mathrm{~h}$ at room temperature followed by staining of the nuclei with Hoechst 33342 (1:2,000, Life Technologies).

TUNEL assays (Millipore) were performed on $12 \mu \mathrm{m}$-thick brain sections of P14 mouse brains post-fixed for $35 \mathrm{~min}$ in ethanol and acetic acid, according to the manufacturer's protocol.

OPCs and OLs were fixed in 2\% PFA, followed by subsequent double immunostaining as previously described ${ }^{64}$. All images were captured using a confocal LSM 510 NLO system or a Nikon Eclipse Ti inverted microscope (Nikon). Representative photographs were obtained with the same exposure setting for control and mutant.

In situ hybridization was performed on $12 \mu \mathrm{m}$-thick brain sections as previously described ${ }^{65,66}$. Probes targeting Plp (Addgene, Cat \#22651) and Pdgfra (kind gift from Charles Stiles) were generated by digesting the plasmids with EcoRI and HindIII, respectively. DIG-labelled RNA probes were generated using Sp6 and T7 polymerase in vitro transcription (Roche Applied Science; DIG RNA labelling kit) as per manufacturer's instructions. Hybridization occurred at $68^{\circ} \mathrm{C}$ and washes at $65^{\circ} \mathrm{C}$. To detect the DIG-labelled probes, the TSA-Plus Cyanine 3 labelling system (Perkin Elmer) was used according to the manufacturer's instructions.

FluoroMyelin Fluorescent Myelin Stains (Life Technologies) were performed on $12 \mu \mathrm{m}$-thick brain sections of P14 and P28 Gpr56 ${ }^{-1-}$ pups and Gpr56 ${ }^{+1-}$ littermate controls according to the manufacturer's protocol. Percentage area myelinated was quantified by outlining the $\mathrm{CC}$ and determining the number of pixels brighter than +10 a.u. (0-255) above background per total number of pixels as previously described ${ }^{65}$ 


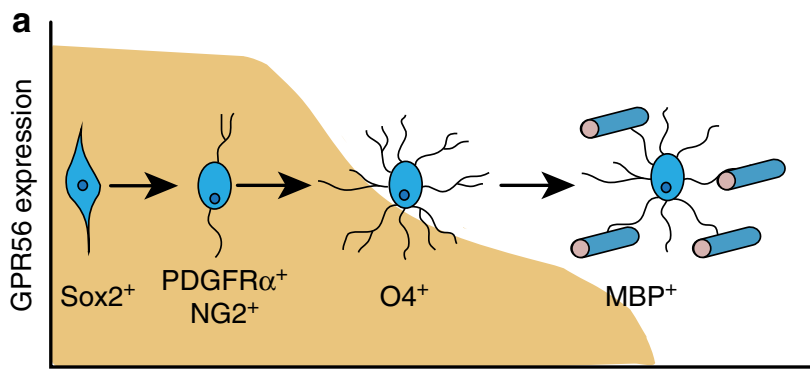

Oligodendrocyte differentiation

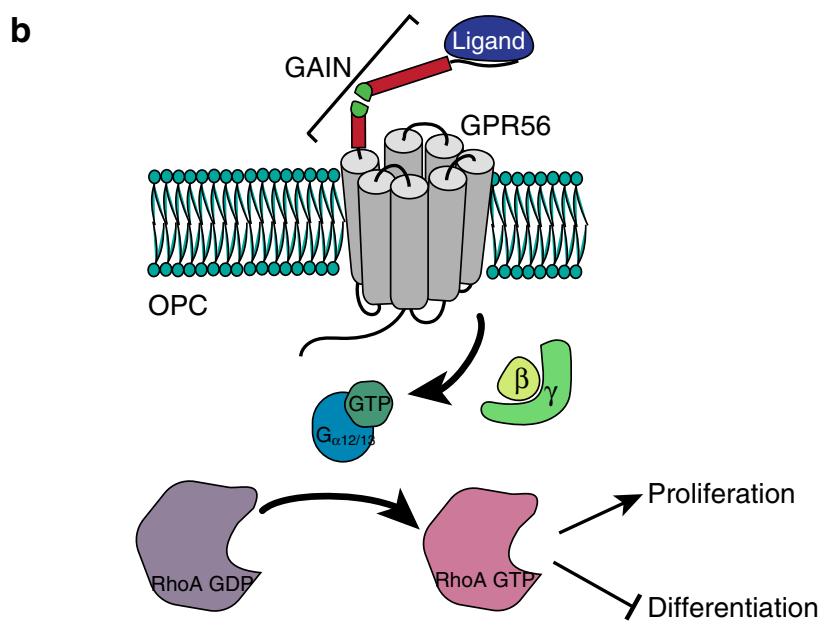

Figure 8 | GPR56 keeps OPCs in a proliferative state. (a) GPR56 is mainly expressed in $\mathrm{NG}^{+} / \mathrm{PDGFR}^{+} \mathrm{OPCs}$. Its expression is downregulated beginning at the immature $\mathrm{O}^{+}{ }^{+} \mathrm{OL}$ stage. The shaded area depicts the developmental stages where GPR56 expression is detected. (b) Model of GPR56 function in OPCs. GPR56, on binding to its unknown ligand, promotes OPC proliferation by activating RhoA.

Tamoxifen treatment and cell cycle exit assay. A solution of $10 \mathrm{mg} \mathrm{ml}^{-1}$ tamoxifen (Sigma; T5648) was prepared in corn oil. Male and female pups were injected intraperitoneally once daily for five consecutive days at $50 \mathrm{mg} \mathrm{kg}^{-1}$ body weight from P10 to P14. Mouse brains were harvested on P21, 7 days after the last tamoxifen injection.

Cell cycle exit assays were performed as previously described ${ }^{67}$. In brief, proliferating cells were labelled with $\operatorname{BrdU}\left(50 \mathrm{mg} \mathrm{kg}^{-1}\right)$ by intraperitoneal injection of male and female P13 Gpr56 ${ }^{-1-}$ pups and their littermate controls. After $24 \mathrm{~h}$, mouse brains were harvested after perfusion, fixed with $4 \%$ PFA, cryoprotected with $30 \%$ sucrose and embedded in OCT. Brain sections were processed for IHC with anti-BrdU and anti-Ki67 antibodies.

Western blot and GTP-Rho pull-down assay. The CCs were dissected under a Leica stereo microscope (MZ 6; Leica Pte), followed by washes in PBS and lysis in ice-cold RIPA buffer (1\% Nonidet P-40, $50 \mathrm{mM}$ Tris pH 7.6, $120 \mathrm{mM} \mathrm{NaCl}, 1 \mathrm{mM}$ EDTA) containing protease inhibitor cocktail set 1 (Calbiochem). The lysates were cleared of insoluble materials by centrifugation at $16,000 \mathrm{~g}$ for $10 \mathrm{~min}$ at $4{ }^{\circ} \mathrm{C}$. Protein concentration was determined by a Bio-Rad protein assay method (Bio$\mathrm{Rad}$ ) according to the manufacturer's protocol, and equal amount of proteins were used for SDS-PAGE and western blot analysis. The GTP-Rho pull-down assay was performed as previously described ${ }^{40}$, using mouse optic nerves of male and female P7 Gpr56 ${ }^{-1-}$ pups and their littermate controls. In brief, P7 mouse optic nerves were pooled according to their genotype into heterozygous and knockout groups. Tissues were grinded as a powder on liquid nitrogen and lysed in $300 \mu \mathrm{l}$ of ice cold RIPA buffer containing protease inhibitors with a cell disruptor for $10 \mathrm{~min}$ and homogenization with syringe needle $26 \mathrm{G}$. An equal amount of total protein was incubated with $60 \mu \mathrm{g}$ GST-RBD beads (Cytoskeleton) at $4^{\circ} \mathrm{C}$ for $90 \mathrm{~min}$. The beads were washed twice with lysis buffer and once with TBS buffer. Bound Rho proteins were eluted by Laemmli sample buffer and detected by western blotting using a mouse monoclonal anti-RhoA antibody (Cytoskeleton).

Transmission electron microscopy. Postnatal male brains were fixed by immersion in mixture of $2.5 \%$ glutaraldehyde and $2 \%$ paraformaldehyde in $0.1 \mathrm{M}$ sodium cacodylate buffer, $\mathrm{pH}$ 7.4. After overnight fixation, tissues were postfixed in $1 \%$ osmium tetroxide, then dehydrated and embedded in Epon-Araldite. Ultrathin sections were cut and stained with uranyl acetate and lead citrate. The samples were observed and photographed using the transmission electron microscope (Tecnai $\mathrm{G}^{2}$ Spirit BioTWIN) at the Harvard Medical School EM Facility. Optic nerves were processed and imaged as described ${ }^{9}$. The photographs were analysed using Image J Software (http://rsb.info.nih.gov/ij/) to calculate g-ratio and axon diameter. G-ratio was calculated as previously described ${ }^{68}$.

OPC cultures. McCarthy-deVellis cultures were used for terminal differentiation experiments (Fig. 6e,f). OPCs were isolated from the forebrains of male and female P1 Gpr56 1 - - pups and their littermate controls after genotyping, as previously described $^{69}$. In brief, dissociated cells from the forebrains of $\mathrm{P} 1$ pups were cultured for 10 days at $37^{\circ} \mathrm{C}$ and $8.5 \% \mathrm{CO}_{2}$ before shaking at 45 r.p.m. and $37^{\circ} \mathrm{C}$ for $1 \mathrm{~h}$ to remove microglia cells. Media was replaced with fresh DMEM/FBS, and the culture was again shaken at 250 r.p.m. and $37^{\circ} \mathrm{C}$ for $18-20 \mathrm{~h}$ to harvest OPCs, followed by incubating on $10 \mathrm{~cm}$ Petri dish for $60 \mathrm{~min}$ at $37^{\circ} \mathrm{C}$ to remove contaminating astrocytes and microglia. Purified OPCs were thoroughly dissociated, plated onto poly-D-lysine-coated coverslips and cultured for 7 days in thyroid hormone containing differentiation medium for terminal differentiation.

O4 panning cultures were performed for proliferation and process elaboration assays. OPCs were isolated from male and female P5-6 Gpr56 ${ }^{+1+}$ or Gpr56 $6^{-1-}$ mouse forebrains, as previously described ${ }^{36,37}$. Immunopanning was carried out using mouse anti-Thy1.2 (Serotec, Cat \#MCA02R) and mouse anti-GalC (Millipore, Cat \#MAB342) for negative selection, followed by mouse anti-O4 AB (O4 hybridoma supernatant) for positive selection. OPCs were released from the $\mathrm{O} 4$ plate by trypsinization and resuspended in media as previously described ${ }^{35}$. Purified OPCs were plated on PDL- or laminin-coated coverslips and cultured in proliferating media containing PDGF-AA and NT-3 (PreproTech) for 4 days or in differentiating medium containing thyroid hormone and B27 for 7 days. Cells were then fixed with $2 \%$ PFA and stained for PDGFR $\alpha$ and Ki67 or for MBP. The area of the myelin sheath of oligodendrocytes was measured using NIS-Elements 3.1 Advanced Research (Nikon) software as previously described ${ }^{59}$ by outlining the myelin sheath area (as indicated in Fig. 6c).

Statistical analysis. For all studies, images were scored blinded to genotype before quantifications. Data are represented as mean \pm s.e.m. GRAPHPAD Prism Software (GraphPad Software) was used to determine statistical significance between genotypes using unpaired $t$-tests or paired $t$-tests, two-tailed and unequal variance depending on animals either being paired before data collection or not. For in vitro culture, animals were paired before isolation of OPCs. Statistical significance between genotypes was determined using paired $t$-tests, two-tailed and unequal variance. Sample size was not pre-determined by statistical methods, but was based on similar studies in the field.

\section{References}

1. Baumann, N. \& Pham-Dinh, D. Biology of oligodendrocyte and myelin in the mammalian central nervous system. Physiol. Rev. 81, 871-927 (2001).

2. Emery, B. Regulation of oligodendrocyte differentiation and myelination. Science 330, 779-782 (2010).

3. Colognato, H. \& Tzvetanova, I. D. Glia unglued: how signals from the extracellular matrix regulate the development of myelinating glia. Dev. Neurobiol. 71, 924-955 (2011).

4. Chun, S. J., Rasband, M. N., Sidman, R. L., Habib, A. A. \& Vartanian, T. Integrin-linked kinase is required for laminin-2-induced oligodendrocyte cell spreading and CNS myelination. J. Cell Biol. 163, 397-408 (2003).

5. Relucio, J., Tzvetanova, I. D., Ao, W., Lindquist, S. \& Colognato, H. Laminin alters fyn regulatory mechanisms and promotes oligodendrocyte development J. Neurosci. 29, 11794-11806 (2009).

6. Relucio, J., Menezes, M. J., Miyagoe-Suzuki, Y., Takeda, S. \& Colognato, H Laminin regulates postnatal oligodendrocyte production by promoting oligodendrocyte progenitor survival in the subventricular zone. Glia 60, 1451-1467 (2012).

7. Langenhan, T., Aust, G. \& Hamann, J. Sticky signaling-adhesion class G protein-coupled receptors take the stage. Sci. Signal 6, re3 (2013).

8. Singer, K., Luo, R., Jeong, S. J. \& Piao, X. GPR56 and the developing cerebral cortex: cells, matrix, and neuronal migration. Mol. Neurobiol. 47, 186-196 (2013).

9. Mogha, A. et al. Gpr126 functions in Schwann cells to control differentiation and myelination via G-protein activation. J. Neurosci. 33, 17976-17985 (2013).

10. Monk, K. R. et al. A G protein-coupled receptor is essential for Schwann cells to initiate myelination. Science 325, 1402-1405 (2009).

11. Shin, D., Lin, S. T., Fu, Y. H. \& Ptacek, L. J. Very large G protein-coupled receptor 1 regulates myelin-associated glycoprotein via Galphas/Galphaqmediated protein kinases A/C. Proc. Natl Acad. Sci. USA 110, 19101-19106 (2013).

12. Piao, X. et al. G protein-coupled receptor-dependent development of human frontal cortex. Science 303, 2033-2036 (2004). 
13. Piao, X. et al. Genotype-phenotype analysis of human frontoparietal polymicrogyria syndromes. Ann. Neurol. 58, 680-687 (2005).

14. Jin, Z. et al. Disease-associated mutations affect GPR56 protein trafficking and cell surface expression. Hum. Mol. Genet. 16, 1972-1985 (2007).

15. Luo, R. et al. A novel GPR56 mutation causes bilateral frontoparietal polymicrogyria. Pediatr. Neurol. 45, 49-53 (2011).

16. Luo, R., Jin, Z., Deng, Y., Strokes, N. \& Piao, X. Disease-associated mutations prevent GPR56-collagen III interaction. PLoS ONE 7, e29818 (2012).

17. Chiang, N. Y. et al. Disease-associated GPR56 mutations cause bilateral frontoparietal polymicrogyria via multiple mechanisms. J. Biol. Chem. 286, 14215-14225 (2011).

18. Ke, N. et al. Biochemical characterization of genetic mutations of GPR56 in patients with bilateral frontoparietal polymicrogyria (BFPP). Biochem. Biophys. Res. Commun. 366, 314-320 (2008).

19. Li, S. et al. GPR56 regulates pial basement membrane integrity and cortical lamination. J. Neurosci. 28, 5817-5826 (2008).

20. Koirala, S., Jin, Z., Piao, X. \& Corfas, G. GPR56-regulated granule cell adhesion is essential for rostral cerebellar development. J. Neurosci. 29, 7439-7449 (2009).

21. Bae, B. I. et al. Evolutionarily dynamic alternative splicing of GPR56 regulates regional cerebral cortical patterning. Science 343, 764-768 (2014).

22. Piao, X. et al. An autosomal recessive form of bilateral frontoparietal polymicrogyria maps to chromosome 16q12.2-21. Am. J. Hum. Genet. 70, 1028-1033 (2002)

23. Chang, B. S. et al. Bilateral frontoparietal polymicrogyria: clinical and radiological features in 10 families with linkage to chromosome 16. Ann. Neurol. 53, 596-606 (2003).

24. Li, G. et al. Regional distribution of cortical interneurons and development of inhibitory tone are regulated by Cxcl12/Cxcr4 signaling. J. Neurosci. 28, 1085-1098 (2008).

25. Hoffmann, S. A. et al. Stem cell factor Sox 2 and its close relative Sox3 have differentiation functions in oligodendrocytes. Development 141, 39-50 (2014).

26. Zhou, Q., Wang, S. \& Anderson, D. J. Identification of a novel family of oligodendrocyte lineage-specific basic helix-loop-helix transcription factors. Neuron 25, 331-343 (2000)

27. Lu, Q. R. et al. Sonic hedgehog-regulated oligodendrocyte lineage genes encoding bHLH proteins in the mammalian central nervous system. Neuron 25, 317-329 (2000).

28. Lu, Q. R. et al. Common developmental requirement for Olig function indicates a motor neuron/oligodendrocyte connection. Cell 109, 75-86 (2002).

29. Kang, S., Fukaya, M., Yang, J., Rothstein, J. \& Bergles, D. NG2 + CNS glial progenitors remain committed to the oligodendrocyte lineage in postnatal life and following neurodegeneration. Neuron 68, 668-681 (2010).

30. Reynolds, R. \& Hardy, R. Oligodendroglial progenitors labeled with the O4 antibody persist in the adult rat cerebral cortex in vivo. J. Neurosci. Res. 47, 455-470 (1997)

31. Barradas, P. C., Vieira, R. S. \& De Freitas, M. S. Selective effect of hypothyroidism on expression of myelin markers during development. J. Neurosci. Res. 66, 254-261 (2001).

32. Zhang, Y. et al. An RNA-sequencing transcriptome and splicing database of glia, neurons, and vascular cells of the cerebral cortex. J. Neurosci. 34, 11929-11947 (2014).

33. Mallon, B. S., Shick, H. E., Kidd, G. J. \& Macklin, W. B. Proteolipid promoter activity distinguishes two populations of NG2-positive cells throughout neonatal cortical development. J. Neurosci. 22, 876-885 (2002)

34. Makinodan, M., Rosen, K. M., Ito, S. \& Corfas, G. A critical period for social experience-dependent oligodendrocyte maturation and myelination. Science 337, 1357-1360 (2012).

35. Dugas, J. C., Tai, Y. C., Speed, T. P., Ngai, J. \& Barres, B. A. Functional genomic analysis of oligodendrocyte differentiation. J. Neurosci. 26, 10967-10983 (2006).

36. Watkins, T., Emery, B., Mulinyawe, S. \& Barres, B. Distinct stages of myelination regulated by gamma-secretase and astrocytes in a rapidly myelinating CNS coculture system. Neuron 60, 555-569 (2008).

37. Wang, S., Sdrulla, A., Johnson, J. E., Yokota, Y. \& Barres, B. A. A role for the helix-loop-helix protein Id2 in the control of oligodendrocyte development. Neuron 29, 603-614 (2001).

38. Belachew, S. et al. Cyclin-dependent kinase-2 controls oligodendrocyte progenitor cell cycle progression and is downregulated in adult oligodendrocyte progenitors. J. Neurosci. 22, 8553-8562 (2002).

39. Jablonska, B. et al. Cdk2 is critical for proliferation and self-renewal of neural progenitor cells in the adult subventricular zone. J. Cell Biol. 179, 1231-1245 (2007).

40. Luo, R. et al. G protein-coupled receptor 56 and collagen III, a receptor-ligand pair, regulates cortical development and lamination. Proc. Natl Acad. Sci. USA 108, 12925-12930 (2011).

41. Yang, L. et al. GPR56 Regulates VEGF production and angiogenesis during melanoma progression. Cancer Res. 71, 5558-5568 (2011).
42. Luo, S., Mao, C., Lee, B. \& Lee, A. S. GRP78/BiP is required for cell proliferation and protecting the inner cell mass from apoptosis during early mouse embryonic development. Mol. Cell Biol. 26, 5688-5697 (2006).

43. Rivers, L. E. et al. PDGFRA/NG2 glia generate myelinating oligodendrocytes and piriform projection neurons in adult mice. Nat. Neurosci. 11, 1392-1401 (2008).

44. Zhu, Q. et al. Genetic evidence that Nkx2.2 and Pdgfra are major determinants of the timing of oligodendrocyte differentiation in the developing CNS. Development 141, 548-555 (2014).

45. Sauvageot, C. M. \& Stiles, C. D. Molecular mechanisms controlling cortical gliogenesis. Curr. Opin. Neurobiol. 12, 244-249 (2002).

46. Parnavelas, J. G. Glial cell lineages in the rat cerebral cortex. Exp. Neurol. 156, 418-429 (1999).

47. Levison, S. W., Chuang, C., Abramson, B. J. \& Goldman, J. E. The migrational patterns and developmental fates of glial precursors in the rat subventricular zone are temporally regulated. Development 119, 611-622 (1993).

48. Zerlin, M., Levison, S. W. \& Goldman, J. E. Early patterns of migration, morphogenesis, and intermediate filament expression of subventricular zone cells in the postnatal rat forebrain. J. Neurosci. 15, 7238-7249 (1995).

49. Bahi-Buisson, N. et al. GPR56-related bilateral frontoparietal polymicrogyria: further evidence for an overlap with the cobblestone complex. Brain 133, 3194-3209 (2010).

50. Jeong, S. J., Li, S., Luo, R., Strokes, N. \& Piao, X. Loss of Col3a1, the gene for Ehlers-Danlos syndrome type IV, results in neocortical dyslamination. PLoS ONE 7, e29767 (2012)

51. Xu, L., Begum, S., Hearn, J. D. \& Hynes, R. O. GPR56, an atypical G protein-coupled receptor, binds tissue transglutaminase, TG2, and inhibits melanoma tumor growth and metastasis. Proc. Natl Acad. Sci. USA 103, 9023-9028 (2006).

52. Fesus, L. \& Piacentini, M. Transglutaminase 2: an enigmatic enzyme with diverse functions. Trends Biochem. Sci. 27, 534-539 (2002).

53. Gaudry, C. A. et al. Cell surface localization of tissue transglutaminase is dependent on a fibronectin-binding site in its N-terminal beta-sandwich domain. J. Biol. Chem. 274, 30707-30714 (1999).

54. Telci, D. et al. Fibronectin-tissue transglutaminase matrix rescues RGDimpaired cell adhesion through syndecan-4 and betal integrin co-signaling. J. Biol. Chem. 283, 20937-20947 (2008).

55. Bailey, C. D. \& Johnson, G. V. Developmental regulation of tissue transglutaminase in the mouse forebrain. J. Neurochem. 91, 1369-1379 (2004)

56. Van Strien, M. E. et al. Tissue transglutaminase activity is involved in the differentiation of oligodendrocyte precursor cells into myelin-forming oligodendrocytes during CNS remyelination. Glia 59, 1622-1634 (2011).

57. Iguchi, T. et al. Orphan G protein-coupled receptor GPR56 regulates neural progenitor cell migration via a G alpha $12 / 13$ and Rho pathway. J. Biol. Chem. 283, 14469-14478 (2008)

58. Paavola, K. J., Stephenson, J. R., Ritter, S. L., Alter, S. P. \& Hall, R. A. The N terminus of the adhesion G protein-coupled receptor GPR56 controls receptor signaling activity. J. Biol. Chem. 286, 28914-28921 (2011).

59. Rajasekharan, S. et al. Netrin 1 and Dcc regulate oligodendrocyte process branching and membrane extension via Fyn and RhoA. Development 136, 415-426 (2009).

60. Rajasekharan, S., Bin, J. M., Antel, J. P. \& Kennedy, T. E. A central role for RhoA during oligodendroglial maturation in the switch from netrin-1-mediated chemorepulsion to process elaboration. J. Neurochem. 113, 1589-1597 (2010).

61. Liang, X., Draghi, N. A. \& Resh, M. D. Signaling from integrins to Fyn to Rho family GTPases regulates morphologic differentiation of oligodendrocytes. J. Neurosci. 24, 7140-7149 (2004).

62. Liu, X. et al. Slit2 regulates the dispersal of oligodendrocyte precursor cells via Fyn/RhoA signaling. J. Biol. Chem. 287, 17503-17516 (2012).

63. Esbenshade, T. A. G Protein-Coupled Receptors as Targets for Drug Discovery (Taylor \& Francis Group, 2006).

64. Jeong, S. J., Luo, R., Li, S., Strokes, N. \& Piao, X. Characterization of G protein-coupled receptor 56 protein expression in the mouse developing neocortex. J. Comp. Neurol. 520, 2930-2940 (2012).

65. Dugas, J. C. et al. Dicer1 and miR-219 Are required for normal oligodendrocyte differentiation and myelination. Neuron 65, 597-611 (2010).

66. Bialas, A. R. \& Stevens, B. TGF-beta signaling regulates neuronal C1q expression and developmental synaptic refinement. Nat. Neurosci. 16, 1773-1782 (2013).

67. Chenn, A. \& Walsh, C. A. Regulation of cerebral cortical size by control of cell cycle exit in neural precursors. Science 297, 365-369 (2002).

68. Roy, K. et al. Loss of erbB signaling in oligodendrocytes alters myelin and dopaminergic function, a potential mechanism for neuropsychiatric disorders. Proc. Natl Acad. Sci. USA 104, 8131-8136 (2007).

69. O’Meara, R., Ryan, S., Colognato, H. \& Kothary, R. Derivation of enriched oligodendrocyte cultures and oligodendrocyte/neuron myelinating co-cultures from post-natal murine tissues. J. Visualiz. Exp. 54, e3324 1-9, doi: $10.3791 / 3324$ (2011). 


\section{Acknowledgements}

We thank Drs Christopher A. Walsh and Jacques Michaud for providing the brain MRI images of a reported BFPP patient; Dr Charles Stiles for anti-Olig2 antibody and the Pdgfr $\alpha$ plasmid for in situ hybridization; Dr J. Bradley Zuchero for consultation on immunopanning of OPCs; Mouse Gene Manipulation Core at Boston Children's Hospital (NIHP30-HD 18655) for their assistance in generating floxed Gpr56 mice. This research was supported in part by NINDS grant R01 NS057536 (X.P.); William Randolph Hearst Fund Award (S.G., R.L., \& S.-J.J.); Leonard and Isabelle Goldenson Research Fellowship (R.L.); NINDS grant F31 NS087801; NINDS grant R01 NS079445 (K.R.M.); and Cerebral Palsy International Research Foundation Award (X.P.).

\section{Author contributions}

S.G. performed and analysed all experiments related to Figs 3, 4c,d, 5a-g, 6, 7d,e, and Supplementary Figs 5, 7a,b as well as assisted all aspects of the data analysis. Y.D. conceived, performed and analysed all experiments related to Figs 1, 2, 4a,b, Supplementary Figs 2, 3, 4a,b and 6. R.L. generated Gpr56-floxed mice, performed and analysed all experiments related to Figs 5 h,i, $7 \mathrm{a}-\mathrm{c}$ and Supplementary Fig. 7. S.-J.J. assisted data analysis on TEM. Y.Y. performed and assisted with the ISH data analysis. S.D.A., A.M., K.R.M. performed the TEM in Fig. 2g. A.R.B., and B.S. assisted on in vitro OPC cultures. M.M. and G.C. helped data acquisition and analysis on TEM and PLP:eGFP. B.S.C. helped on Supplementary Fig. 1. X.P. conceived the experiments and oversaw all aspects of data analyses. The paper was written by S.G., Y.D. and X.P.

\section{Additional information}

Supplementary Information accompanies this paper at http://www.nature.com/ naturecommunications

Competing financial interests: The authors declare no competing financial interests.

Reprints and permission information is available online at http://npg.nature.com/ reprintsandpermissions/

How to cite this article: Giera, S. et al. The adhesion G protein-coupled receptor GPR56 is a cell-autonomous regulator of oligodendrocyte development. Nat. Commun. 6:6121 doi: $10.1038 /$ ncomms7121 (2015).

(c) (i) This work is licensed under a Creative Commons Attribution 4.0 International License. The images or other third party material in this article are included in the article's Creative Commons license, unless indicated otherwise in the credit line; if the material is not included under the Creative Commons license, users will need to obtain permission from the license holder to reproduce the material. To view a copy of this license, visit http://creativecommons.org/licenses/by/4.0/ 